\title{
FACTORES ASOCIADOS A VELOCIDAD DE MARCHA LENTA EN ADULTOS MAYORES DE UN DISTRITO EN LIMA, PERÚ
}

\author{
Gabriela Rodríguez ${ }^{1, a}$, Daniella Burga-Cisneros ${ }^{1, a}$, Gabriela Cipriano1,a , Pedro J. Ortiz ${ }^{1,2, b, c}$, Tania Tello ${ }^{1,2, d, e}$ \\ Paola Casas ${ }^{1,2, d}$, Elizabeth Aliaga ${ }^{1,2, d}$, Luis F. Varela ${ }^{1,2, f, g}$
}

\section{RESUMEN}

\begin{abstract}
Objetivos. Determinar los factores asociados a velocidad de marcha lenta en adultos mayores de la comunidad residentes en un distrito de Lima, Perú. Materiales y métodos. Estudio de análisis de base de datos secundario. Se incluyó a los adultos mayores de 60 años y se excluyó aquellos con condiciones que no garantizaban la evaluación de la marcha. La variable dependiente fue la velocidad de marcha lenta, menor a $1 \mathrm{~m} / \mathrm{s}$, y las variables independientes fueron sociodemográficas, clínicas y de valoración geriátrica integral. Se calcularon las razones de prevalencia (RP) crudas y ajustadas con intervalos de confianza al 95\% (IC95\%). Resultados. Se incluyeron 416 adultos mayores, el rango de edad fue de 60 a 99 años y un $41 \%$ presentaba velocidad de marcha lenta. Se encontró que los factores asociados a una velocidad de marcha lenta en adultos mayores de la comunidad son el sexo femenino (RP 1,45, IC95\%: 1,13-1,88), la edad mayor de 70 años (RP 1,73, IC95\%: 1,30-2,30), un menor grado de instrucción (RP. 2.07, IC95\%: 1,20-3,55), la presencia de problema socio familiar (RP 1,66, IC95\%: 1,08-2,54), la presencia de diabetes mellitus (RP 1,35, IC95\%: 1,01-1,80) y de depresión (RP: 1.41, IC95\%: 1,02-1,95). Conclusiones. Los factores modificables asociados a una velocidad de marcha lenta en el adulto mayor en la comunidad son tanto clínicos como sociofamiliares, susceptibles de intervención desde etapas precoces en el curso de la vida.
\end{abstract}

Palabras clave: Velocidad de marcha, adulto mayor, comunidad. (Fuente: DeCS BIREME)

\section{FACTORS ASSOCIATED WITH SLOW WALKING SPEED IN OLDER ADULTS OF A DISTRICT IN LIMA, PERU}

\begin{abstract}
Objectives. To determine the factors associated with slow walking speed in older adults living in a district of Lima, Peru. Materials and methods. Analysis of secondary data. Adults older than 60 years were included in the study, while adults with physical conditions who did not allow the evaluation of the walking speed were excluded. The dependent variable was slow walking speed (less than $1 \mathrm{~m} / \mathrm{s}$ ), and the independent variables were sociodemographic, clinical, and geriatric data. Raw and adjusted prevalence ratios (PR) were calculated with $95 \%$ confidence intervals $(95 \% \mathrm{Cl})$. Results. The study sample included 416 older adults aged 60 to 99 years, and $41 \%$ of the participants met the slow walking speed criterion. The factors associated with slow walking speed in this sample were female gender $(\mathrm{PR}, 1.45 ; 95 \% \mathrm{Cl}, 1.13-1.88)$, age $>70$ years $(\mathrm{PR}, 1.73 ; 95 \% \mathrm{Cl}, 1.30$ 2.30), lower level of education ( $\mathrm{PR}, 2.07,95 \% \mathrm{Cl}, 1.20-3.55)$, social-familial problems (PR, 1.66; 95\% Cl, 1.08-2.54), diabetes mellitus (PR, 1.35; 95\% Cl, 1.01-1.80), and depression (PR, 1.41; $95 \% \mathrm{Cl}, 1.02-1.95)$. Conclusions. The modifiable factors associated with slow walking speed in older adults included clinical and social-familial problems, and these factors are susceptible to interventions from the early stages of life.
\end{abstract}

Key words: Gait speed, older people, community. (Source: MeSH NLM).

\section{INTRODUCCIÓN}

En el contexto de la evaluación geriátrica, la medición de la velocidad de la marcha representa una prueba rápida, de bajo costo y reproducible ${ }^{(1)}$, que traduce fuerza, coordinación y balance como resultado del buen funcionamiento de los múltiples órganos y sistemas involucrados en su desempeño ${ }^{(2)}$. El presentar una velocidad de la marcha lenta resulta ser un indicador independiente de mortalidad en adultos mayores ${ }^{(3)}$, habiéndose demostrado que valores menores a $1 \mathrm{~m} / \mathrm{s}$, predicen eventos adversos tales como mortalidad, admisiones hospitalarias, discapacidad, limitación en la función de los miembros inferiores y caídas ${ }^{(2-5)}$. Una detección oportuna permite prevenir

Facultad de Medicina, Universidad Peruana Cayetano Heredia. Lima, Perú.

Instituto de Gerontología, Universidad Peruana Cayetano Heredia. Lima, Perú.

Estudiante de Medicina; ${ }^{\mathrm{b}}$ Médico internista; ${ }^{\mathrm{c}}$ Magíster en Medicina; ${ }^{\mathrm{d}}$ Médico geriatra; ${ }^{\mathrm{e}}$ Magíster en Geriatría y Gerontología; ${ }^{\mathrm{f}}$ Médico internista y geriatra;

${ }^{\mathrm{g}}$ Doctor en Medicina

Recibido: 27/07/2017 Aprobado: 30/11/2017 En línea: 07/12/2017

Citar como: Rodríguez G, Burga-Cisneros D, Cipriano G, Ortiz PJ, Tello T, Casas P, et al. Factores asociados a velocidad de marcha lenta en adultos mayores de un distrito en Lima, Perú. Rev Peru Med Exp Salud Publica. 2017;34(4): 619-26.doi: 10.17843/rpmesp.2017.344.3025 
el rápido deterioro de la salud del adulto mayor con intervenciones que logran una reducción del riesgo de muerte en un $18 \%$, y por tal relevancia se ha propuesto que la velocidad de la marcha sea considerada un sexto signo vital en adultos mayores, tal como la medición de la presión arterial o de la frecuencia cardiaca ${ }^{(1)}$.

Diversos estudios epidemiológicos sobre los factores contribuyentes y asociados a una velocidad de la marcha lenta muestran información que varía según el escenario y la población estudiada ${ }^{(6-8)}$. Estos hallazgos sugieren la existencia de determinantes ajenos a la propia edad que de estar presentes puedan contribuir a detectar precozmente una velocidad de la marcha lenta en adultos mayores aparentemente sanos; por ello es necesario identificar aquellos factores que, al no depender de la edad, sean modificables y permitan intervenir en mejorar la velocidad de la marcha.

El objetivo del presente estudio es determinar los factores asociados a una velocidad de la marcha lenta en adultos mayores que viven en la comunidad, lo cual permitirá identificar aquella población en riesgo y vulnerable en nuestro medio, caracterizado por una alta tasa de envejecimiento y un inadecuado acceso a los servicios de salud ${ }^{(9)}$.

\section{MATERIALES Y MÉTODOS}

\section{DISEÑO}

Estudio de análisis secundario de la base de datos "Perfil clínico, funcional y sociofamiliar del adulto mayor de San Martín de Porres" (10), un estudio transversal de adultos mayores de 60 años que se realizó en el distrito de San Martín de Porres, el segundo más poblado de Lima, con una población de personas mayores de 60 años de 70,568 que representa el $10 \%$ de la población total del distrito y un índice de envejecimiento (la razón entre el número de adultos mayores de 60 años sobre el número de niños y jóvenes menores de 18 años) de 0,37. Los datos fueron recolectados de enero a mayo del año 2013.

\section{POBLACIÓN}

Se incluyó a los adultos mayores de 60 años a más de la comunidad del distrito de San Martín de Porres que pudiesen caminar sin ningún tipo de ayuda o asistencia. El criterio de inclusión consistió en la inclusión al estudio original ${ }^{(10)}$ y los criterios de exclusión estuvieron basados en las recomendaciones propuestas por Fried et al. ${ }^{(11)} \mathrm{y}$ de Montero-Odasso ${ }^{(12)}$ que garantizaron una evaluación objetiva de la velocidad de la marcha. Por ello, para efectos del presente estudio, se excluyeron a aquellos participantes con dependencia severa y total para las actividades de la vida diaria de acuerdo a la evaluación funcional con el Índice de Barthel (13), aquellos con deterioro cognitivo

\section{MENSAJES CLAVE}

Motivación para realizar el estudio. Una velocidad de la marcha lenta predice eventos adversos en la salud del adulto mayor y no se conocen cuáles son los factores que están asociados en adultos mayores aparentemente sanos que viven en la comunidad.

Principales hallazgos. Se halló que ser mujer, tener más edad, un bajo nivel de instrucción, problemas sociales, diabetes y depresión están asociados a la velocidad de marcha lenta en adultos mayores que viven en un distrito de Lima, Perú.

Implicancias. Estas características deberán ser usadas en atención primaria para identificar un perfil de riesgo en la población adulta mayor y así dirigir la aplicación de intervenciones para mejorarla.

moderado a severo según el test de Pfeiffer ${ }^{(14)}$, aquellos con cualquier condición neurológica que altere la marcha y a aquellos quienes no tuvieron medición de la velocidad de marcha. El universo de la población estuvo representado por 546 adultos mayores, de los cuales 45 no aceptaron su inclusión al estudio, restando 501 como total. La selección de los participantes en el presente estudio se muestra en la Figura 1.

\section{MUESTREO}

Para tener una idea de la población adulta mayor total del distrito y su distribución por sectores, se utilizó la cobertura de pacientes adultos mayores atendidos en los diferentes establecimientos de salud del Ministerio de Salud en el distrito. Posteriormente, el distrito fue subdividido en ocho sectores de acuerdo a la localización geográfica de los 16 establecimientos de atención primaria y un hospital general. Se asignaron números a los bloques de cada sector de acuerdo a una tabla de números aleatorios. El equipo de trabajo de campo compuesto por cuatro geriatras fue informado diariamente de los bloques seleccionados a visitar hasta completar el tamaño de muestra por cada sector. Las viviendas seleccionadas para evaluar dentro de cada bloque fueron elegidas por los evaluadores de forma aleatoria según la tabla de números aleatorios y se entrevistaron a los adultos mayores que residían en cada vivienda. Si no hubo habitantes adultos mayores en la vivienda seleccionada se evaluó la vivienda contigua. Todos los adultos mayores que vivían en cada hogar y que eran elegibles fueron invitados a participar, mediante 44 salidas de campo en las que participaron cuatro médicos geriatras capacitados.

\section{TAMAÑO MUESTRAL}

El tamaño de la muestra del estudio original fue calculado en 450 adultos mayores, y se alcanzó una muestra total de 501 participantes luego del trabajo de campo. Para el presente estudio un análisis post-hoc fue realizado buscando diferentes muestras según los resultados 
del análisis multivariado. Para detectar una diferencia de proporciones de adultos mayores con velocidad de marcha lenta, según el sexo se requerían 224 adultos mayores. Calculando el tamaño para detectar diferencias con respecto a la presencia de depresión, el tamaño de muestra requerido fue de 65 adultos mayores. Por ello, el presente estudio tiene el poder suficiente para determinar las correlaciones entre una velocidad de marcha lenta y las variables indicadas.

\section{DATOS Y MEDICIONES}

Cuatro geriatras recolectaron la información mediante una entrevista personal y un cuestionario estructurado donde se registró las variables demográficas, socioeconómicos, la evaluación física, afectiva, cognitiva, social, síndromes y problemas geriátricos. Se evaluaron finalmente los datos antropométricos y de velocidad de la marcha.

\section{VARIABLE DEPENDIENTE}

La velocidad de la marcha fue determinada por el tiempo requerido por el participante para caminar a la velocidad de una marcha usual, por una distancia de $8 \mathrm{~m}$ de una distancia total de $10 \mathrm{~m}$ sin considerar el primer y el último metro recorrido sobre una superficie plana. El tiempo más rápido de dos mediciones seguidas fue elegido como valor final y una velocidad de la marcha lenta será considerada en valores menores a $1 \mathrm{~m} / \mathrm{s}^{(2,5)}$.

\section{VARIABLES INDEPENDIENTES}

Se incluyeron las variables de acuerdo a los instrumentos validados y descritos en el estudio original, entre ellas, la edad y su clasificación en tres grupos etarios (60 a 69 años, 70 a 79 años y mayores de 80 años), sexo, variables sociodemográficas como: estado civil (con pareja, sin pareja); grado de instrucción (sin instrucción, instrucción escolar, instrucción superior); ser jefe de familia; situación económica familiar durante la infancia (mala, regular, buena); estado de salud en la infancia (mala, buena, excelente); momentos de hambre en la infancia; tipo de familia (nuclear, extendida, sin núcleo y unipersonal); poseedor de seguro de salud; actividades en tiempo libre (remuneradas, domésticas o recreacionales); la valoración sociofamiliar a través de la escala de Guijón, estableciéndose tres categorías (entorno social adecuado, riesgo y problema social) y la disfunción familiar evaluada mediante el APGAR familiar (no disfunción familiar, moderada disfunción o severa disfunción familiar).

Las variables clínicas incluyeron la autopercepción de salud (muy buena/buena, regular, muy mala/mala), la presencia de enfermedades crónicas por autorreporte (hipertensión arterial, diabetes, osteoartritis, osteoporosis, enfermedades pulmonares crónicas), multimorbilidad (dos o más enfermedades crónicas), el autorreporte de consumo de alcohol y tabaco, la polifarmacia por el uso de tres a más medicamentos, la presencia de síndromes y problemas geriátricos (caídas, déficit visual o auditivo, trastornos del sueño), depresión evaluada por la escala reducida de depresión geriátrica (no depresión, depresión leve y depresión establecida), la evaluación nutricional evaluada de acuerdo al índice de masa corporal (IMC) y el Mini Nutritional Assessment ${ }^{(15)}$.

\section{ANÁLISIS ESTADÍSTICO}

Se presentan los resultados mediante frecuencias y porcentajes para las variables categóricas, y medias y desviación estándar para las continuas y se realizó el análisis de asociaciones de variables mediante el test de chi cuadrado y análisis de varianza.

Para evaluar los factores asociados a una velocidad de la marcha lenta se calcularon las razones de prevalencia (RP) crudas y ajustadas con los intervalos de confianza al 95\% (IC95\%) usando la regresión de Poisson con varianza robusta, dado que reduce problemas de convergencia en estudios transversales con variable resultado dicotómico a diferencia de la regresión logística ${ }^{(16)}$. Para el análisis multivariado se incluyó a las variables que tuvieron significancia clínica en el análisis bivariado y se consideró un $p<0,05$ como significativo. El valor crudo muestra los valores del RP de forma independiente y el RP ajustado, según la interrelación con las demás variables. Las variables consideradas en el ajuste fueron edad, sexo, dependencia funcional, deterioro cognitivo y la presencia de caídas. Los análisis fueron realizados con el programa STATA 14 (StataCorp, 2015. College Station, TX, USA).

\section{CONSIDERACIONES ÉTICAS}

El estudio original fue aprobado por el Comité Institucional de Ética en Investigación de la Universidad Peruana Cayetano Heredia, así como el presente estudio. Toda la información del estudio se mantuvo bajo códigos sin identificación de los participantes, quienes autorizaron el uso de los datos para futuros estudios como este y todos firmaron el consentimiento informado.

\section{RESULTADOS}

Se incluyó la información de 416 adultos mayores del estudio original, el rango de edad fue de 60 a 99 años y el mayor porcentaje se encontraba entre 60 y 69 años (52,6\%). Según el sexo, 259 (62,3\%) fueron mujeres. La media de la velocidad de la marcha fue de 1,02 m/s, DE: 0,01 y la proporción de personas con velocidad de marcha lenta fue de $41 \%(n=171)$.

Se encontró medias más bajas y proporciones más altas de velocidad de marcha lenta en adultos mayores del sexo femenino, edad mayor de 70 años, menor grado 
de instrucción, haber tenido momentos de hambre en la infancia, no ser jefe de familia, participar en actividades recreacionales no laborales, tener problema sociofamiliar, una mala autopercepción de su salud, la presencia de hipertensión arterial, de diabetes mellitus, al consumo de alcohol, la ausencia de multimorbilidad, tener dependencia funcional, malnutrición, caídas, somnolencia, polifarmacia, deterioro cognitivo y depresión. Las características sociodemográficas y del estado de salud de los adultos mayores según la velocidad de marcha se muestran en la Tabla 1 y 2.

Se encontró que los factores asociados a una velocidad de marcha lenta en adultos mayores de la comunidad fueron el sexo femenino (RP:1,45, IC95\%: 1,13-1,88), la edad mayor a 70 años (RP: 1,73, IC95\%: 1,30-2,30), un menor grado de instrucción (RP 2,07, IC95\%: 1,20-3,55), la presencia de problema socio familiar (RP 1,66, IC95\%: 1,08-2,54), de diabetes mellitus (RP 1,35, IC95\%: 1,01-1,80) y depresión establecida (RP 1,41, IC95\%: 1,02-1,95) como se muestra en la Tabla 3.

\section{DISCUSIÓN}

Diferentes estudios revelan que existen determinantes físicos, tanto modificables como no modificables, en el enlentecimiento de la marcha en adultos mayores ${ }^{(6-8)}$, sin embargo, en el presente estudio se demuestra que una velocidad de marcha lenta en adultos mayores de la comunidad está asociada también a un factor social, lo cual revela la importancia del efecto del entorno en el que se desenvuelve el adulto mayor sobre el desempeño físico y la génesis de la futura discapacidad y dependencia.

En este contexto, algunos estudios también han demostrado la asociación entre una velocidad de marcha lenta y las inequidades sociales, el ambiente o entorno en el que viven, el soporte social que reciben y la cohesión y relaciones sociales que son a su vez determinantes directos del estado de salud de las personas mayores ${ }^{(17-19)}$. De la misma, se ha demostrado que las desventajas socioeconómicas en la adultez y la tercera edad condicionan tasas elevadas de enfermedades crónicas, morbilidad y discapacidad, eventos finales que son precedidos por condiciones subclínicas como deterioro funcional, un bajo nivel de capacidad física y, muy precozmente, por una velocidad de la marcha lenta ${ }^{(20-22)}$.

En el Perú, un país en vías de desarrollo, el 22,9\% de la población vive en condiciones de pobreza ${ }^{(23)}$, lo cual trae como consecuencia un menor acceso a la educación, menores recursos para un desarrollo social y, final e indirectamente, el deterioro en el estado de salud. Estas condiciones suponen el desarrollo de eventos adversos para la salud si no se identifica e interviene precozmente, brindando un mejor soporte y acceso a redes sociales más que la sola implementación de un apoyo económico para la población adulta mayor como se viene realizando actualmente sin que estas medidas conlleven a una mejoría en su salud física ${ }^{(24)}$.

Con respecto a los determinantes físicos no modificables se encontró asociación entre el sexo femenino y el incremento de la edad con la velocidad de marcha lenta, hallazgo que ha sido ampliamente demostrado ${ }^{(6-8,25)}$. Esta fuerte asociación se basa en la alteración progresiva, conforme avanza la edad, del tamaño y fuerza muscular, de la composición corporal y la capacidad aeróbica, importantes determinantes de la marcha ${ }^{(8)}$. Asimismo, se han descrito valores inferiores en el reflejo y fuerza en mujeres adultas mayores en comparación con hombres ${ }^{(26)}$, así como en la fuerza muscular de las extremidades superiores e inferiores, en la capacidad de realizar ejercicio y en la cantidad de masa magra en la mujer adulta mayor ${ }^{(25,26)}$.

Desde el punto de vista clínico, la diabetes tipo 2 es un factor asociado y demostrado que afecta la velocidad de la marcha pues la función de caminar requiere de la contribución de varios sistemas y, siendo la diabetes una condición de afectación multisistémica, se produce un deterioro progresivo de diversos órganos y sistemas indispensables para una correcta caminata ${ }^{(27)}$. Un estudio demostró que la diabetes estaba asociada a una menor densidad y calidad muscular y menor fuerza en la rodilla y el tobillo ${ }^{(28)}$ y también a una menor distancia de recorrido en 6 min ${ }^{(29)}$. Asimismo, se considera que la presencia del estado proinflamatorio como consecuencia de la resistencia a la insulina, producirá una reducción en la fuerza y poder muscular, lo cual, aunado a la neuropatía periférica, explicarían aún más la presencia de una velocidad de la marcha lenta en adultos mayores diabéticos ${ }^{(30)}$.

La población estudiada fue extraída de la comunidad, y con una deambulación sin asistencia, por lo que no se incluyó adultos mayores con diabetes que haya producido daño secuelar a nivel de miembros inferiores o sobre la capacidad cardiaca, respiratoria o neurológica que altere la marcha. Es decir, la propia condición de tener diabetes produciría alteraciones físicas subclínicas que enlentecen la marcha sin que esta sea reflejo de alguna secuela de la enfermedad ${ }^{(27)}$.

Con respecto a la depresión, el cual fue un factor asociado con una velocidad de marcha lenta en este estudio, estudios previos demuestran que los síntomas de depresión predicen la incidencia del deterioro de la velocidad de la marcha y viceversa, con algunos factores asociados intervinientes como sexo femenino, deterioro cognitivo, comorbilidad, discapacidad, bajo nivel socioeconómico y número de hospitalizaciones ${ }^{(31)}$. Otro estudio encontró que una velocidad de marcha lenta en la línea de base predijo síntomas depresivos incidentes tanto en hombres como mujeres ${ }^{(32)}$. 
Tabla 1. Características sociodemográficas asociadas a una velocidad de la marcha lenta en adultos mayores, 2013

\begin{tabular}{|c|c|c|c|}
\hline \multirow{2}{*}{ Característica } & \multirow{2}{*}{$\begin{array}{c}\text { Velocidad de marcha }<1 \mathrm{~m} / \mathrm{s}^{*} \\
\mathrm{n}=171\end{array}$} & \multicolumn{2}{|l|}{ Velocidad de marcha $\geq 1 \mathrm{~m} / \mathrm{s}$} \\
\hline & & $n=245$ & Valor de $p$ \\
\hline & $\mathrm{n}(\%)$ & $\mathrm{n}(\%)$ & \\
\hline \multicolumn{4}{|l|}{ Sexo } \\
\hline Masculino & $52(33,1)$ & $105(66,9)$ & \\
\hline Femenino & $119(45,9)$ & $140(54,1)$ & 0,013 \\
\hline \multicolumn{4}{|l|}{ Grupos etarios (años) } \\
\hline 60 a 69 & $58(26,5)$ & $161(73,5)$ & \\
\hline 70 a 79 & $61(47,3)$ & $68(52,7)$ & $<0,001$ \\
\hline 80 a 99 & $52(76,5)$ & $16(23,5)$ & $<0,001$ \\
\hline \multicolumn{4}{|l|}{ Grado de instrucción } \\
\hline Instrucción superior & $12(15,0)$ & $68(85,0)$ & \\
\hline Instrucción escolar & $147(46,3)$ & $171(53,8)$ & $<0,001$ \\
\hline Sin instrucción & $12(66,7)$ & $6(33,3)$ & $<0,001$ \\
\hline \multicolumn{4}{|l|}{ Estado civil } \\
\hline Con pareja & $101(38,3)$ & $163(61,7)$ & \\
\hline Sin pareja & $70(46,1)$ & $82(53,9)$ & 0,115 \\
\hline \multicolumn{4}{|c|}{ Situación económica familiar durante la infancia } \\
\hline Buena & $53(43,8)$ & $68(56,2)$ & \\
\hline Regular & $82(36,4)$ & $143(63,6)$ & 0,175 \\
\hline Mala & $36(41,4)$ & $34(48,6)$ & 0,302 \\
\hline \multicolumn{4}{|c|}{ Estado de salud durante la infancia } \\
\hline Excelente & $35(42,7)$ & $47(57,3)$ & \\
\hline Buena & $120(40,1)$ & $179(59,9)$ & 0,674 \\
\hline Mala & $16(45,7)$ & $19(54,3)$ & 0,760 \\
\hline \multicolumn{4}{|c|}{ Momentos de hambre durante la infancia } \\
\hline No & $99(37,4)$ & $166(62,6)$ & \\
\hline Sí & $72(47,7)$ & $79(52,3)$ & 0,037 \\
\hline \multicolumn{4}{|l|}{ Jefe de familia } \\
\hline Sí & $69(33,2)$ & $139(66,8)$ & 0,001 \\
\hline No & $102(49,0)$ & $106(51,0)$ & \\
\hline \multicolumn{4}{|l|}{ Tipo de familia } \\
\hline Nuclear & $116(38,9)$ & $182(61,1)$ & \\
\hline Extendida & $38(49,3)$ & $39(50,7)$ & 0,082 \\
\hline Sin núcleo & $2(40,0)$ & $3(60,0)$ & 0,961 \\
\hline Unipersonal & $15(41,7)$ & $21(58,3)$ & 0,746 \\
\hline \multicolumn{4}{|c|}{ Lugar de atención ante problema de salud } \\
\hline Establecimiento de salud & $169(41,5)$ & $238(58,5)$ & \\
\hline No busca atención & $2(22,2)$ & $7(77,8)$ & 0,319 \\
\hline \multicolumn{4}{|l|}{ Portador de seguro de salud } \\
\hline Sí & $115(42,4)$ & $156(57,6)$ & \\
\hline No & $56(38,6)$ & $89(61,4)$ & 0,457 \\
\hline \multicolumn{4}{|l|}{ Actividades en tiempo libre } \\
\hline Remunerada & $42(34,7)$ & $79(65,3)$ & \\
\hline Doméstica & $89(37,9)$ & $146(62,1)$ & 0,562 \\
\hline Recreacional & $16(66,7)$ & $8(33,3)$ & 0,001 \\
\hline \multicolumn{4}{|l|}{ Situación social } \\
\hline Entorno adecuado & $56(32,0)$ & $119(68,0)$ & \\
\hline Entorno en riesgo & $97(45,5)$ & $116(54,5)$ & 0,008 \\
\hline Problema socio familiar & $18(64,3)$ & $10(35,7)$ & $<0,001$ \\
\hline \multicolumn{4}{|l|}{ Disfunción familiar } \\
\hline No disfunción & $143(42,3)$ & $195(57,7)$ & \\
\hline Disfunción moderada & $21(38,9)$ & $33(61,1)$ & 0,74 \\
\hline Disfunción severa & $7(29,2)$ & $17(70,8)$ & 0,25 \\
\hline
\end{tabular}

* Marcha lenta 
Tabla 2. Características del estado de salud asociados a una velocidad de la marcha lenta en adultos mayores,2013

\begin{tabular}{|c|c|c|c|}
\hline \multirow{3}{*}{ Característica } & Velocidad de marcha $<1 \mathrm{~m} / \mathrm{s}^{*}$ & Velocidad de marcha $\geq 1 \mathrm{~m} / \mathrm{s}$ & \multirow{3}{*}{ Valor de $\mathrm{p}$} \\
\hline & $\mathrm{n}=171$ & $n=245$ & \\
\hline & $\mathrm{n}(\%)$ & $\mathrm{n}(\%)$ & \\
\hline \multicolumn{4}{|c|}{ Autorreporte del estado de salud } \\
\hline Muy buena/buena & $35(33,9)$ & $68(66,1)$ & \\
\hline Regular & $102(39,8)$ & $154(60,2)$ & 0,312 \\
\hline Muy mala/mala & $34(59,7)$ & $23(40,3)$ & 0,001 \\
\hline \multicolumn{4}{|l|}{ Índice de masa corporal } \\
\hline Normal & $25(42,4)$ & $34(57,6)$ & \\
\hline Sobrepeso & $75(41,2)$ & $107(58,8)$ & 0,874 \\
\hline Bajo peso & $34(34,0)$ & $66(66,0)$ & 0,286 \\
\hline Obesidad & $37(49,3)$ & $38(50,7)$ & 0,428 \\
\hline \multicolumn{4}{|l|}{ Hipertensión arterial } \\
\hline No & $90(35,6)$ & $163(64,4)$ & \\
\hline Sí & $81(49,7)$ & $82(50,3)$ & 0,004 \\
\hline \multicolumn{4}{|l|}{ Diabetes mellitus } \\
\hline No & $138(38,7)$ & $219(61,3)$ & \\
\hline Sí & $33(55,9)$ & $26(44,1)$ & 0,006 \\
\hline \multicolumn{4}{|l|}{ Enfermedad cardiovascular } \\
\hline No & $150(39,8)$ & $227(60,2)$ & \\
\hline Sí & $21(58,8)$ & $18(46,2)$ & 0,061 \\
\hline \multicolumn{4}{|c|}{ Enfermedad pulmonar crónica } \\
\hline No & $157(40,5)$ & $231(59,5)$ & \\
\hline Sí & $14(50,0)$ & $14(50,0)$ & 0,29 \\
\hline \multicolumn{4}{|l|}{ Dislipidemia } \\
\hline No & $130(40,8)$ & $189(59,2)$ & \\
\hline Sí & $41(42,3)$ & $56(57,7)$ & 0,27 \\
\hline \multicolumn{4}{|l|}{ Enfermedad reumatológica } \\
\hline No & $105(38,2)$ & $170(61,8)$ & \\
\hline Sí & $66(46,8)$ & $75(53,2)$ & 0,085 \\
\hline Consumo de alcohol & & & \\
\hline No consume & $162(43,8)$ & $208(56,2)$ & \\
\hline Consume & $9(19,6)$ & $37(80,4)$ & 0,008 \\
\hline Consumo de tabaco & & & \\
\hline No fuma & $160(42,3)$ & $217(57,7)$ & \\
\hline Fuma & $11(28,2)$ & $28(71,8)$ & 0,12 \\
\hline Multimorbilidad & & & \\
\hline Sí & $92(35,1)$ & $170(64,9)$ & \\
\hline No & $79(51,3)$ & $75(48,7)$ & 0,001 \\
\hline Estado funcional & & & \\
\hline Independiente & $123(37,3)$ & $207(62,7)$ & \\
\hline Dependiente parcial & $48(55,8)$ & $38(44,2)$ & 0,001 \\
\hline Estado de nutrición (MNA) & & & \\
\hline Normal & $118(37,8)$ & $194(62,2)$ & \\
\hline Con riesgo & $41(48,2)$ & $44(51,8)$ & 0,069 \\
\hline Malnutrido & $12(63,2)$ & $7(36,8)$ & 0,007 \\
\hline SÍNDROMES Y PROBLEN & & & \\
\hline Caídas & & & \\
\hline No & $96(34,7)$ & $181(65,3)$ & \\
\hline Sí & $75(53,9)$ & $64(46,1)$ & $<0,001$ \\
\hline Déficit visual & & & \\
\hline No & $36(35,3)$ & $66(64,7)$ & \\
\hline Sí & $135(43,0)$ & $179(57,0)$ & 0,17 \\
\hline Déficit auditivo & & & \\
\hline No & $89(37,4)$ & $149(62,6)$ & \\
\hline Sí & $82(46,1)$ & $96(53,9)$ & 0,075 \\
\hline Insomnio de conciliación & & & \\
\hline No & $108(40,6)$ & $158(59,4)$ & \\
\hline Sí & $63(42,0)$ & $87(58,0)$ & 0,781 \\
\hline Insomnio de mantenimient & & & \\
\hline No & $91(41,0)$ & $131(59,0)$ & \\
\hline Sí & $80(41,2)$ & $114(58,8)$ & 0,959 \\
\hline Somnolencia & & & \\
\hline No & $87(36,1)$ & $154(63,9)$ & \\
\hline Sí & $84(48,0)$ & $91(52,0)$ & 0,015 \\
\hline Polifarmacia & & & \\
\hline No & $130(38,1)$ & $211(61,9)$ & \\
\hline Sí & $41(54,7)$ & $34(45,3)$ & 0,008 \\
\hline Estado cognitivo & & & \\
\hline Sin deterioro & $143(38,1)$ & $232(61,9)$ & \\
\hline Con deterioro leve & $28(68,3)$ & $13(31,7)$ & $<0,001$ \\
\hline Depresión & & & \\
\hline Normal & $132(37,9)$ & $216(62,1)$ & \\
\hline Riesgo de depresión & $22(53,7)$ & $19(46,3)$ & 0,031 \\
\hline Depresión establecida & $62(63,0)$ & $10(37,0)$ & 0,002 \\
\hline
\end{tabular}

*Marcha lenta. MNA: Mini-Nutritional Assessment 
Tabla 3. Factores asociados a una velocidad de la marcha lenta en adultos mayores de un distrito en Lima, Perú (2013)

\begin{tabular}{|c|c|c|c|c|}
\hline Variables & RP crudo (IC 95\%)* & Valor de $p$ & RP ajustado (IC 95\%)** & Valor de $p$ \\
\hline \multicolumn{5}{|l|}{ Sexo } \\
\hline Masculino & 1 & & 1 & \\
\hline Femenino & $1,39(1,07-1,79)$ & 0,013 & $1,45(1,13-1,88)$ & 0,003 \\
\hline \multicolumn{5}{|l|}{ Grupos etarios (años) } \\
\hline 60 a 69 & 1 & & 1 & \\
\hline 70 a 79 & $1,79(1,34-2,38)$ & $<0,001$ & $1,73(1,30-2,30)$ & $<0,001$ \\
\hline 80 a 99 & $2,89(2,23-3,73)$ & $<0,001$ & $2,77(2,09-3,69)$ & $<0,001$ \\
\hline \multicolumn{5}{|l|}{ Grado de instrucción } \\
\hline Instrucción superior & 1 & & 1 & \\
\hline Instrucción escolar & $3,08(1,80-5,26)$ & $<0,001$ & $2,07(1,20-3,55)$ & 0,008 \\
\hline Sin instrucción & $4,44(2,40-8,23)$ & $<0,001$ & $1,80(0,91-3,54)$ & 0,090 \\
\hline \multicolumn{5}{|l|}{ Situación social } \\
\hline Entorno adecuado & 1 & & 1 & \\
\hline Entorno en riesgo & $1,42(1,10-1,85)$ & 0,008 & $1,13(0,87-1,50)$ & 0,354 \\
\hline Problema socio familiar & $2,01(1,41-2,85)$ & $<0,001$ & $1,66(1,08-2,54)$ & 0,020 \\
\hline \multicolumn{5}{|l|}{ Diabetes mellitus } \\
\hline No & 1 & & 1 & \\
\hline Sí & $1,45(1,11-1,88)$ & 0,006 & $1,35(1,01-1,80)$ & 0,045 \\
\hline \multicolumn{5}{|l|}{ Depresión } \\
\hline Normal & 1 & & 1 & \\
\hline Riesgo de depresión & $1,41(1,03-1,94)$ & 0,031 & $1,24(0,92-1,66)$ & 0,165 \\
\hline Depresión establecida & $1,66(1,21-2,28)$ & 0,002 & $1,41(1,02-1,95)$ & 0,037 \\
\hline
\end{tabular}

${ }^{*}$ Regresión de Poisson simple con varianza robusta

**Regresión de Poisson múltiple con varianza robusta; el modelo se generó con todas las variables que mostraron diferencia estadísticamente significativa en el análisis crudo y ajustado para edad, sexo, dependencia funcional, deterioro cognitivo y presencia de caídas.

Entre las limitaciones de nuestro estudio resaltan su naturaleza transversal, lo cual impide determinar asociación de tipo causal; la información recabada fue realizada por autorreporte lo cual pudo generar el sesgo de recuerdo, limitante que es notoria en adultos mayores y que pudiese influenciar los resultados; no se ha podido evaluar el efecto de tener dos personas dentro del mismo hogar pues la proporción fue menor al $8 \%$ de la muestra y, finalmente, si bien la muestra se calculó para lograr un nivel de inferencia significativo, el número representa menos del $1 \%$ de la población total de adultos mayores del distrito. Su principal fortaleza radica en el tamaño significativo de la muestra y el haberse realizado en una población de adultos mayores que viven en la comunidad.

Se concluye que los factores asociados a una velocidad de marcha lenta en el adulto mayor en la comunidad sirven en atención primaria para identificar un perfil de riesgo en la población adulta mayor y así dirigir la aplicación de intervenciones para mejorarla. Los factores depresión y diabetes mellitus merecen recibir una atención no solo orientada a mejorar los objetivos propios de cada condición, sino también a mejorar la velocidad de marcha y la capacidad física del adulto mayor. El grado de instrucción y el problema social, que requiere enfocarse con mediciones más precisas y específicas, deberán ser foco de intervención desde etapas precoces en el curso de vida de la población como medida de salud pública para reducir su efecto deletéreo en la salud futura de las personas adultas mayores.

Contribuciones de los autores: todos los autores participaron en la concepción y el diseño del estudio; TT, PC, y EA participaron en la recolección de los datos y PJO realizó el análisis de los datos. Todos los autores participaron en la interpretación de los resultados, en la redacción del artículo, su revisión crítica y en la aprobación de la versión final a publicar.

Fuentes de financiamiento: el presente estudio ha sido autofinanciado. El estudio original de donde se extrajo la información fue financiado por el Fondo Cooperante de la Universidad Peruana Cayetano Heredia.

Conflictos de interés: los autores declaran no tener conflictos de interés.

\section{REFERENCIAS BIBLIOGRÁFICAS}

1. Middleton A, Fritz S, Lusardi M. Walking speed: the functional vital sign. J Aging Phys Act. 2015;23(2):314-22.

2. Pamoukdjian F, Paillaud E, Zelek L, Laurent M, Lévy V, Landre T, et al. Measurement of gait speed in older adults to identify complications associated with frailty: A systematic review. J Geriatr Oncol. 2015;6(6):484-96.

3. Abellan van Kan G, Rolland Y, Andrieu $\mathrm{S}$, Bauer J, Beauchet $\mathrm{O}$, Bonnefoy $\mathrm{M}$, et al. Gait speed at usual pace as a predictor of adverse outcomes in community-dwelling older people an International Academy on Nutrition and Aging (IANA)Task Force. J Nutr Health Aging. 2009;13:881-9.

4. Pérez-Zepeda M, Sánchez-Garrido N, González-Lara, M, Gutiérrez-Robledo 
M. Sarcopenia prevalence using simple measurements and population-based cutoff values. J Frailty Aging 2015; 4(3): 139-43.

5. Cesari M, Kritchevsky S, Penninx B, Nicklas B, Simonsick E, Newman A, et al. Prognostic value of Usual Gait Speed in Well-Functioning Older People Results from the Health, Aging and Body Composition Study. J Am Geriatr Soc. 2005;53(10):1675-1680.

6. Dumurgier J, Elbaz A, Ducimetiere P, Tavernier B, Alperovitch A, Tzourio C. Slow walking speed and cardiovascular death in well-functioning older adults: prospective cohort study. BMJ. 2009;339:b4460.

7. Ruggero C, Bilton T, Teixeira L, Ramos J, Alouche S, Dias R, et al. Gait speed correlates in a multiracial population of community-dwelling older adults living in Brazil: a cross-sectional population-based study. BMC Public Health. 2013;13:182.

8. Fiser W, Hays N, Rogers S, Kajkenova O, Williams A, Evans C, et al. Energetics of Walking in Elderly People: Factors Related to Gait Speed. J Gerontol A Biol Sci Med Sci. 2010;65A(12):1332-7.

9. Varela L. Desarrollando respuestas integradas en los sistemas de salud de una población en rápido envejecimiento. INTRA III. Lima: UPCH-OPS/OMS; 2005.

10. Varela-Pinedo L, Chávez-Jimeno H, TelloRodríguez T, Ortiz-Saavedra P, GálvezCano M, Casas-Vasquez P, et al. Clinical, functional and older socio-familiar profile of the community in a district of Lima, Peru. Rev Peru Med Exp Salud Publica. 2015;32(4):709.

11. Fried LP, Tangen CM, Walston J, Newman AB, Hirsch C, Gottdiener J, et al. Frailty in older adults: evidence for a phenotype. J Gerontol A Biol Sci Med Sci. 2001; 56(3):M146-56.

12. Montero-Odasso M, Schapira M, Soriano ER, Varela M, Kaplan R, Camera LA, et al. Gait velocity as a single predictor of adverse events in healthy seniors aged 75 years and older.J Gerontol A Biol Sci Med Sci. 2005;60(10):1304-9.

13. Mahoney FI, Barthel DW. Functional Evaluation: The Barthel Index. Md State Med J 1965; 14:61-5.

14. Martínez de la Iglesia J, Dueñas Herrero R, Onís Vilches MC, Aguado Taberné C, Albert Colomerc C, Luque Luque
R. Spanish language adaptation and validation of the Pfeiffer's questionnaire (SPMSQ) to detect cognitive deterioration in people over 65 years of age. Med Clin (Barc) 2001;117(4):129-34.

15. Guigoz Y, Vellas B, Garry PJ. Assessing the nutritional status of the elderly: The Mini Nutritional Asssessment as part of the geriatric evaluation. Nutr Rev 1996;54(1 Pt 2):S59-65

16. Coutinho LM, Scazufca M, Menezes PR. Methods for estimating prevalence ratios in cross-sectional studies. Rev Saude Publica. 2008 Dec;42(6):992-8

17. McCulloch A. Social environments and health: cross sectional national survey. BMJ. 2001;323(7306):208-9.

18. Woo J, Goggins W, Sham A, Ho SC. Social determinants of frailty. Gerontology. 2005;51(6):402-8.

19. Quispe R, Benziger CP, Bazo-Alvarez JC, Howe LD, Checkley W, Gilman $\mathrm{RH}$, et al. The Relationship Between Socioeconomic Status and CV Risk Factors: The CRONICAS Cohort Study of Peruvian Adults. Glob Heart. 2016;11(1):121-130.e2.

20. Casale-Martínez RI, Navarrete-Reyes AP, Avila-Funes JA. Social determinants of frailty in elderly Mexican communitydwelling adults. J Am Geriatr Soc. 2012;60(4):800-2.

21. Becerra MB, Herring P, Marshak HH, Banta JE. Social Determinants of Physical Activity Among Adult Asian-Americans: Results from a Population-Based Survey in California. J Immigr Minor Health. 2015;17(4):1061-9

22. Plouvier S, Carton M, Cyr D, Sabia S, Leclerc A, Zins M, et al. Socioeconomic disparities in gait speed and associated characteristics in early old age. BMC Musculoskelet Disord. 2016;17:178.

23. Olivera J, Clausen J. Las características del adulto mayor peruano y las políticas de protección social. Economía 2014; 37(73): 75- 113.

24. Gertler P, Galiani S. Evaluación de Impacto del Programa Pensión 65: Nueva evidencia causal de las pensiones no contributivas en Perú. Ministerio de Economía y Finanzas, Lima. 2016.71pp.

25. Fitzpatrick AL, Buchanan CK, Nahin RL, DeKosky ST, Atkinson HH, Carlson $\mathrm{MC}$, et al. Associations of gait speed and other measures of physical function with cognition in a healthy cohort of elderly persons. J Gerontol A Biol Sci Med Sci. 2007;62(11):1244-51.

26. Izawa KP, Watanabe $S$, Hirano $Y$, Matsushima S, Suzuki T, Oka K, et al. Gender-related differences in maximum gait speed and daily physical activity in elderly hospitalized cardiac inpatients: a preliminary study. Medicine (Baltimore). 2015;94(11):e623.

27. Inzitari M, Calle A, Esteve A, Casas A, Torrents N, Martínez N. Do you measure gait speed in your daily clinical practice? A review. Rev Esp Geriatr Gerontol 2017;52:35-43

28. Volpato S, Bianchi L, Lauretani F, Lauretani F, Bandinelli S, Guralnik, et al. Role of muscle mass and muscle quality in the association between diabetes and gait speed. Diabetes Care. 2012;35(8):1672-9.

29. Stewart T, Caffrey DG, Gilman RH, Mathai SC, Lerner A, Hernandez A, et al. Can a simple test of functional capacity add to the clinical assessment of diabetes? Diabet Med. 2016;33(8):1133-9.

30. Kalyani RR, Tra Y, Yeh HC, Egan JM, Ferrucci L, Brancati FL. Quadriceps Strength, Quadriceps Power, and Gait Speed in Older U.S. Adults with Diabetes: Results from the National Health and Nutrition Examination Survey (NHANES), 1999-2002. J Am Geriatr Soc. 2013;61(5):769-75.

31. Duran-Badillo T, Aguilar RM, Martínez ML, Gutiérrez G, Vásquez L, Salazar BC. Asociación de velocidad de marcha y síntomas depresivos en adultos mayores de una comunidad urbano-marginal. Desarrollo Cientif Enferm. 2011;19(2):44-7.

32. Sanders JB., Bremmer MA, Deeg DJ, Beekman AT. Do Depressive Symptoms and Gait Speed Impairment Predict Each Other's Incidence? A 16-Year Prospective Study in the Community. J Am Geriatr Soc. 2012;60(9):1673-80.

\section{Correspondencia: Pedro J. Ortiz}

Dirección: Instituto de Gerontología, Universidad Peruana Cayetano Heredia. Av. Honorio Delgado 430. San Martin de Porres. Lima, Perú.

Teléfono: 3190007

Correo electrónico:pedro.ortiz@upch.pe 